\title{
Punicalagin Suppresses LPS-induced Inflammatory Responses in Murine Macrophages via JAK/STAT Signaling Pathway and Zymosan-induced Mice Paw Edema
}

\author{
Huan Yan ${ }^{1,2}$, Abilaiti Gulimire ${ }^{2,3}$, Junyu Zhu4, Huaping Liang ${ }^{4}$, Zhengyi Gu²,3* \\ ${ }^{1}$ College of Public Health, Xinjiang Medical University, Urumqi, CHINA. \\ ${ }^{2}$ Xinjiang Institute of Materia Medica, Urumqi, CHINA. \\ ${ }^{3}$ College of Pharmacy, Xinjiang Medical University, Urumqi, CHINA. \\ ${ }^{4}$ Department of The Field Surgery, Institute of The Third Affiliated Hospital of Army Medical University, \\ National Key Laboratory of Trauma, Burn and Compound Injury, Chongqing, CHINA.
}

\begin{abstract}
Aim: To investigate the anti-inflammatory activity of punicalagin (PUN) on mouse macrophages and the underlying mechanisms. Materials and Methods: Mouse primary peritoneal macrophages (PMs) were treated with lipopolysaccharide (LPS; $10 \mu \mathrm{g} / \mathrm{ml}$ ) and/ or PUN $(50 \mu \mathrm{M})$ for 4,8 and $12 \mathrm{hr}$. The expression levels of inducible nitric oxide synthase (iNOS), interleukin (IL)-1 $\beta$, IL-6, IL-12P40 and CCL- 2 mRNAs were analyzed by qRT-PCR. Total iNOS, MAPK and JAK/STAT protein levels were measured by Western blotting and the secreted levels of IL-1 $\beta$, IL- 6, IL-12P40 and IL-1270 by ELISA. Acute paw edema was induced in mice with zymosan and the animals were treated with high $(50 \mathrm{mg} / \mathrm{kg})$, medium $(25 \mathrm{mg} / \mathrm{kg})$ and low $(12.5 \mathrm{mg} / \mathrm{kg})$ doses of PUN. The levels of IL-1 3 , IL-6, CCL-2 and other related inflammatory factors and chemokines in the mouse paw tissue homogenates were detected by ELISA. Results: Compared to the LPS-treated cells, PUN significantly decreased the levels of inflammatory cytokines and other factors $(P<0.05)$. In addition, high dose of PUN down regulated p-ERK, p-JAK1, p-STAT1 and p-STAT3, without significantly affecting p-p38 and p-JNK. Finally, acute paw edema in the mouse model was alleviated within $12 \mathrm{~h}$ of high-dose PUN administration, which also inhibited inflammatory cytokines and chemokines. Conclusion: PUN inhibits macrophage-mediated inflammation and the secretion of inflammatory cytokines/chemokines by targeting the ERK/MAPK and JAK/STAT signaling pathways.
\end{abstract}

Key words: Punicalagin, LPS-induced Inflammatory Responses, Macrophages, JAK/STAT, Paw edema.

\section{INTRODUCTION}

Punicalagin (PUN) is the main active ingredient in pomegranate peel extract and has anti-inflammatory, antioxidant, antiviral, anti-tumor, antibacterial and other pharmacological effects. ${ }^{1-3}$ The mechanistic basis of its anti-inflammatory action is the inhibition of the NF- $x \mathrm{~B}$ signaling pathway via blocking phosphorylation of mitogenactivated protein kinase (MAPK), p38, c-Jun amino terminal kinase (JNK) and extracellular protein kinase (ERK). ${ }^{4}$ Macrophages are the primary immune cells involved in triggering inflammation ${ }^{5,6}$ and are regulated by multiple signaling pathways, ${ }^{7,8}$ including the JAK/ STAT signaling pathway that is activated in response to various external stimuli. ${ }^{9}{ }^{90}$ The inflammatory response is driven by several cytokines that are secreted from immune cells and act on the effect or cells through intracellular pathways, thereby affecting cellular activation, proliferation and survival. For instance, macrophages secrete various inflammatory factors such as IL-1 $\beta$, IL-6, iNOS, IL-12P40, IL-12P70 and CCL-2
Submission Date: 18-08-2020; Revision Date: 15-12-2020; Accepted Date: 11-03-2021

DOI: 10.5530/ijper.55.2.93 Correspondence: Mr. Zhengyi Gu ${ }^{2}$ Xinjiang Institute of Materia Medica, Urumqi, China; ${ }^{3}$ College of Pharmacy, Xinjiang Medical University, Urumqi, CHINA.

Phone no: +86 13899857669 Email id: zhengyi087@126. com

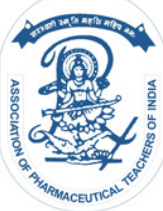

www.ijper.org 
during acute inflammation. ${ }^{11,12}$ The aim of our study was to assess the anti-inflammatory effects of PUN on lipopolysaccharide (LPS)-stimulated primary murine peritoneal macrophages (PMs) and in a zymosan-induced model of paw edema and acute inflammation. ${ }^{13-15}$

\section{MATERIALS AND METHODS}

\section{Reagents}

Punicalagin $(>98 \%)$ and LPS were purchased from Sigma (St.Louis, MO, USA). RPMI 1640 was obtained from GibcoBRL (Gaithersburg, MD, USA). ELISA kits for IL-1 $\beta$, IL-6, CCL-2 and CCL-4 were purchased from $\mathrm{R}$ and D Systems (Minneapolis, MN, USA) and that for IL-12p40 and IL-12p70 from DaKeWe (ShenZhen, China). Antibodies targeting iNOS, p38, p-p38, ERK, p-ERK, JNK, p-JNK, JAK1, p-JAK1, STAT1, STAT3, SHP2, p-SHP and SOCS3 were purchased from Cell Signaling Technology (Danvers, MA, USA).

\section{Cell culture}

Primary PMs were extracted from female C57BL/6 mice aged 8 weeks. The cells were cultured in RPMI-1640 medium supplemented with 10\% FBS and antibiotics $\left(100 \mathrm{U} / \mathrm{ml}\right.$ penicillin and $100 \mathrm{U} / \mathrm{ml}$ streptomycin) at $37^{\circ} \mathrm{C}$ in a humidified incubator under $5 \% \mathrm{CO}_{2}$. For LPS/PUN treatment, the PMs were seeded in 6-well plates at the density of $2 \times 10^{6}$ cells/well and treated with $10 \mu \mathrm{g} / \mathrm{ml}$ LPS and/or 50 $\mu \mathrm{M}$ PUN ( $n=3$ each) for 4, 8, 12 and $24 \mathrm{hr}$ as appropriate.

\section{Quantitative RT-PCR Analysis}

Total RNA was extracted from the suitably treated cells, reverse transcribed and amplified using SYBR Premix (TaKaRa) on a BioRad CFX96 cycler. Primer sequences are Table 1.

\section{Enzyme-Linked Immunosorbent Assay (ELISA)}

Supernatants were collected from the suitably treated cells after $12 \mathrm{~h}$ and $24 \mathrm{~h}$ of culture and the levels of IL-1 $\beta$, IL-6, IL-12p40, IL-12p70, CCL-2 and CCL-4 were measured using ELISA kits according to the manufacturer's instructions.

\section{Western Blotting}

The suitably treated PMs were washed twice with ice-cold PBS and lyzed in $200 \mu 1$ RIPA lysis buffer supplemented with protease inhibitors on ice for $10 \mathrm{~min}$. The homogenates were gently scraped from the plate, transferred to $1.5 \mathrm{~mL}$ pre-cooled EP tubes and placed on ice for $15 \mathrm{~min}$ while overtaxing every $5 \mathrm{~min}$. The cell extracts were centrifuged at $13,000 \times \mathrm{g}$ at $4^{\circ} \mathrm{C}$ for $10 \mathrm{~min}$ and the supernatants were collected. Following quantification with the BCA kit, equal amounts of protein per sample were separated on 10\% Tris-Glycine extended polyacrylamide gels. Densitometric analysis of the bands was performed using Image J.

\section{Establishment of zymosan-induced mouse paw edema model}

Acute inflammation was modeled in vivo by inducing edema in the left hind paws mice through a single injection of $50 \mu \mathrm{l}$ zymosan $(1 \%)$. Four hours later, the animals were injected intraperitoneally with 25,50 and $100 \mathrm{mg} / \mathrm{kg}$ of PUN or $5 \mathrm{mM}$ dexamethasone (positive control). Paw edema was monitored over a period of $24 \mathrm{~h}$ and the animals were euthanized. The paw tissues were harvested and then stored at $-80^{\circ} \mathrm{C}$. IL-1b, IL-6, CCL-2 and CCL- 4 content in the paw tissue homogenates were analyzed by ELISAkits.

\section{Statistical Analysis}

Statistical analysis was performed using GraphPad Prism 7.0 software. Data was expressed as mean \pm SEM from three experiments and compared using unpaired t-test. $P<0.05$ was considered statistically significant.

\section{RESULTS}

\section{PUN inhibits the inflammatory response in LPS- stimulated macrophages}

LPS challenge significantly increased the production of iNOS mRNA and protein in PMs compared to that in the untreated controls, which was inhibited by PUN in a time-dependent manner (Figure 1A, B). Consistent with this, LPS also markedly increased the mRNA levels of

\begin{tabular}{|c|c|c|}
\hline Gene & Reverse primer $\left(5^{\prime} \rightarrow \rightarrow^{\prime}\right)$ & Forward primer $\left(5^{\prime} \rightarrow \rightarrow^{\prime}\right)$ \\
\hline iNOS & GTGGACGGGTCGATGTCAC & GTTCTCAGCCCAACAATACAAGA \\
\hline IL-1 $\beta$ & ATCTTTTGGGGTCCGTCAACT & GCAACTGTTCCTGAACTCAACT \\
\hline IL-6 & CTCATTTCCACGATTTCCCAG & ACCACGGCCTTCCCTACTTC \\
\hline CCL-2 & GCATTAGCTTCAGATTTACGGGT & TTAAAAACCTGGATCGGAACCAA \\
\hline IL-12b & GACTGTAGTAGGTAGGGTGAGG & CATACCGTTGGGACAGACCG \\
\hline$\beta$-actin & CTCTCAGCTGTGGTGGTGAA & AGCCATGTACGTAGCCATCC \\
\hline
\end{tabular}


several inflammatory cytokines. PUN intervention rapidly decreased the levels of CCL-2 and IL-12P40 (Figure 2C, D) and that of IL- $1 \beta$ and IL- 6 after $8 \mathrm{~h}$ and $12 \mathrm{~h}$ (Figure $2 \mathrm{~A}, \mathrm{~B}$ ). Furthermore, LPS stimulation increased secretion of the above inflammatory cytokines was inhibited by PUN with the exception of IL-6 (Figure 3).

\section{PUN inhibits ERK/MAPK and JAK/SAT pathways in LPS-stimulated PMs}

To further elucidate the mechanism underlying the antiinflammatory effect of PUN, we analyzed the levels of
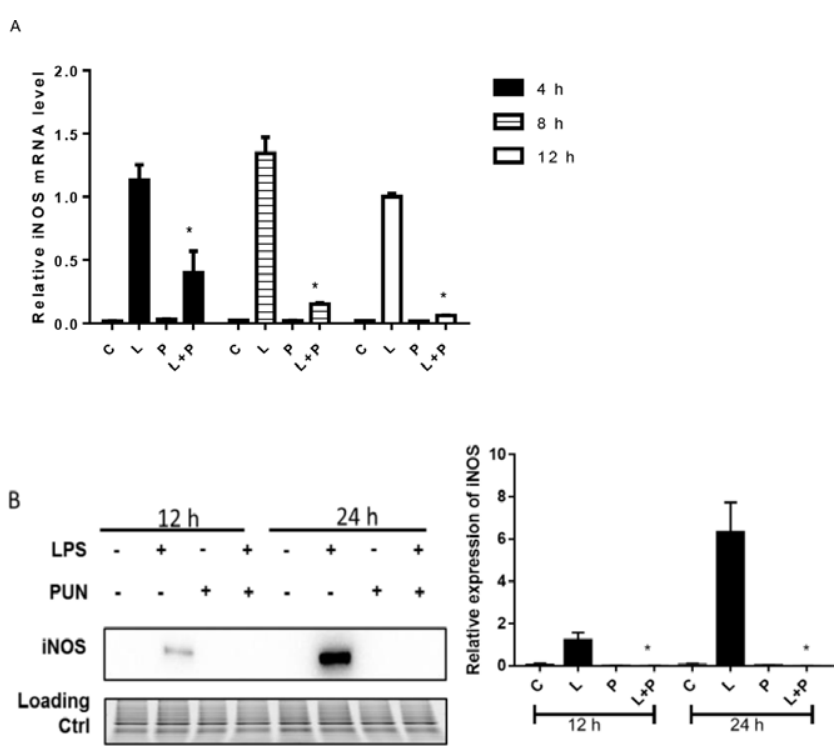

Figure 1: Effect of PUN on iNOS mRNA and protein in PMs. A. RT-PCR assay showing iNOS mRNA levels in PMs treated with $10 \mu \mathrm{g} / \mathrm{ml}$ LPS and $50 \mu \mathrm{M}$ PUN for 4,8 and $12 \mathrm{~h}$. B. Immunoblot and bar graph showing iNOS protein levels in the above treated cells. $p<0.05$ compared with the LPS-treated group.
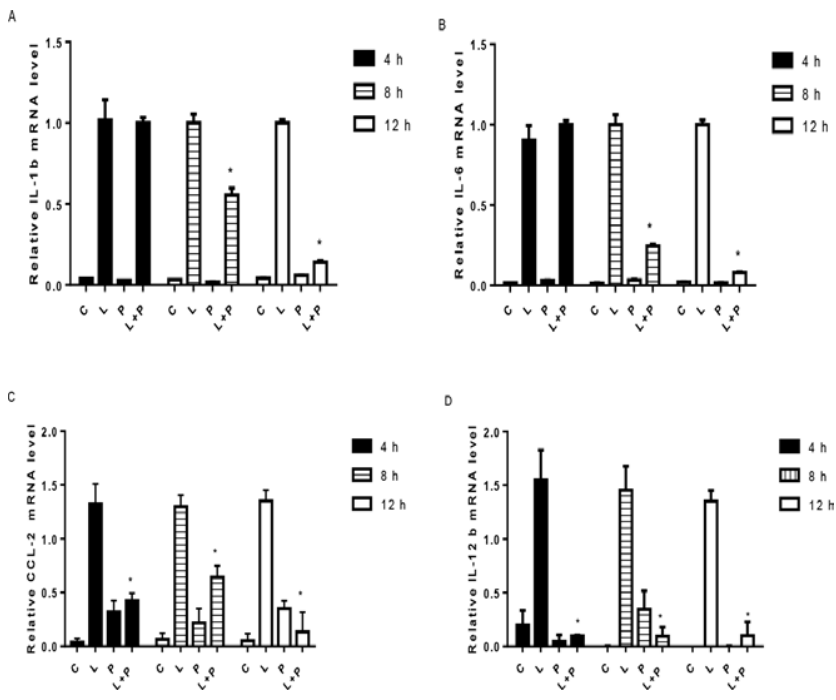

Figure 2: Effect of PUN on LPS-induced proinflammatory cytokine mRNAs. The levels of IL-1b (A), IL-6 (B). CCL-2 (C) and IL-12b(D) mRNAs in PMs treated with $10 \mu \mathrm{g} / \mathrm{ml}$ LPS and $50 \mu \mathrm{M}$ PUN for 4, 8 and $12 \mathrm{~h} .{ }^{*} p<0.05$ compared with the LPS-treated group.
ERK/MAPK pathway mediators in the LPS-stimulated cells.LPS induced phosphorylation of ERK, p38 and JNK in the murine PMs (Figure 4A): While PUN significantly inhibited p-ERK expression (Figure 4B), it had no effect on the total and phosphorylated JNK and p38 levels (Figure 4C, D). Since the JAK/STAT pathway is also involved in the process of inflammation, we analyzed the levels of JAK1, STAT1/3 and the negative regulatory
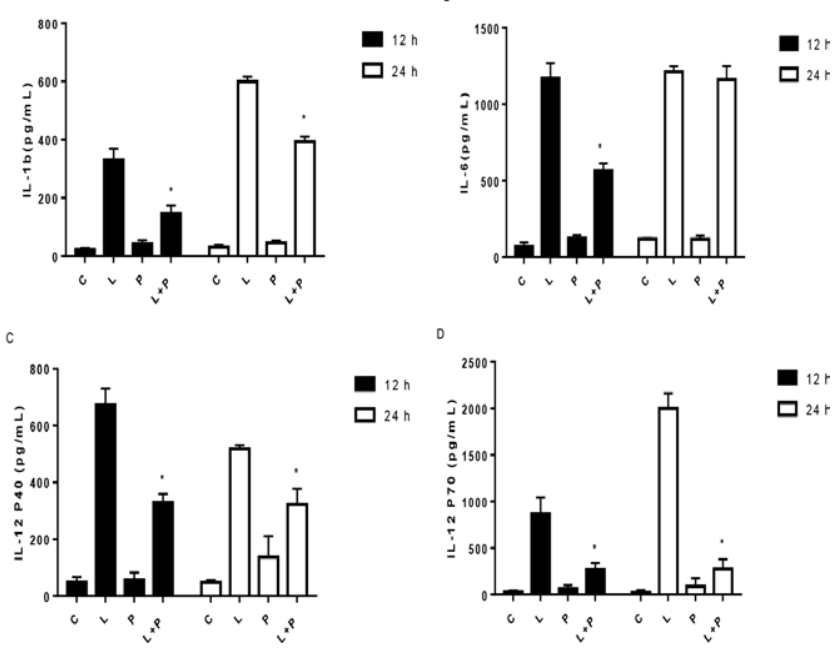

Figure 3: Effect of PUN on LPS-induced secretion of pro-inflammatory cytokines. IL-1 $\beta$ (A), IL-6 (B), IL-12P40 (C) and IL-12P70 (D) levels in the supernatants of PMs treated with $10 \mu \mathrm{g} / \mathrm{ml}$ LPS and 50 $\mu \mathrm{M}$ PUN for 12 and $24 \mathrm{~h}$. ${ }^{*} p<0.05$ compared with the LPS-treated group.
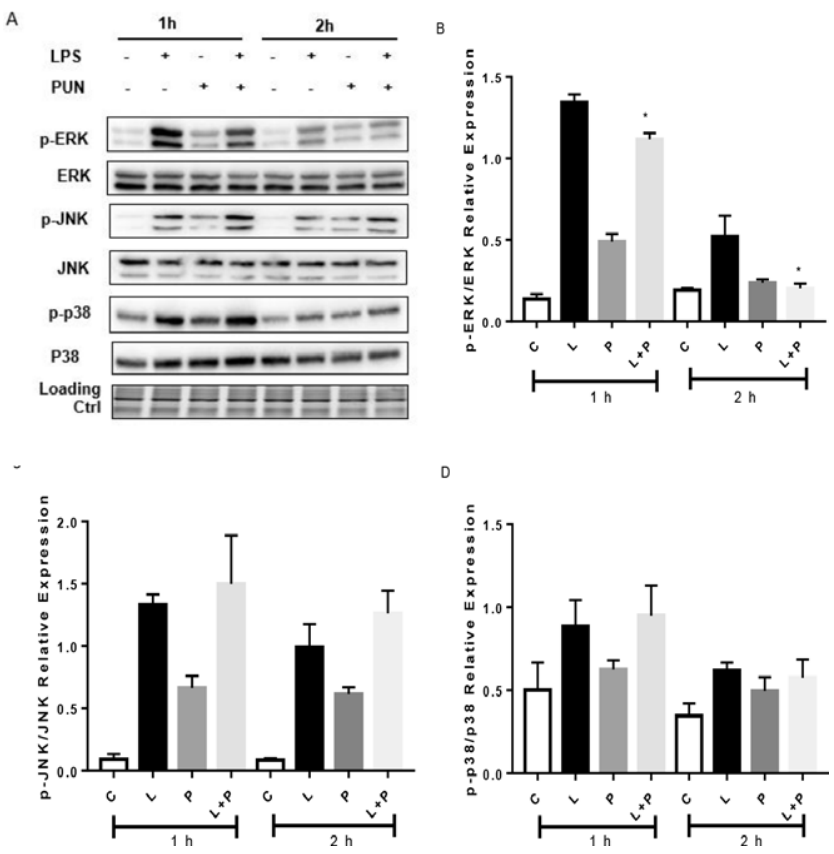

Figure 4: The effect of PUN on LPS-induced phosphorylation of MAPKs in PMs cells. Phosphorylation levels of p38, JNK and ERK in PMs treated with $10 \mu \mathrm{g} / \mathrm{ml}$ LPS and $50 \mu \mathrm{M}$ PUN for the indicated time points. ${ }^{*} p<0.05$ compared with the LPS-treated group. 
proteins $\mathrm{SOCS}_{3}$ and SHP. As shown in Figure 5A, LPS significantly increased JAK1, STAT1 and STAT3 phosphorylation, which was prevented by PUN treatment (Figure 5B-D). In addition, PUN slightly increased the levels of SOCS3 (Figure 5E) but had no effect on SHP2 (Figure 5E, F). Taken together, PUN inhibits the inflammatory response in PMs by blocking both the ERK/MAPK and JAK/STAT pathways.

\section{Effect of PUN on Zymosan-induced mouse paw edema}

The in vivo effects of PUN were assessed in a murine zymosan-induced paw edema model. An acute inflammatory reaction was triggered in the left hind paw within $1 \mathrm{~h}$ of zymosan injection, characterized by redness and edema. As shown in Figure 6A, high-dose PUN alleviated swelling within $12 \mathrm{~h}$ compared to that in the untreated model group, whereas the low and medium doses of PUN had minimal effect even after 24h. Consistent with this, the paw homogenates of the untreated model showed high levels of IL-6, CCL-2 and CCL-4 and all but IL-6 were reduced significantly after high-dose PUN intervention (Figure 6B-E). Thus, PUN can relieve symptoms of acute localized inflammation in vivo.

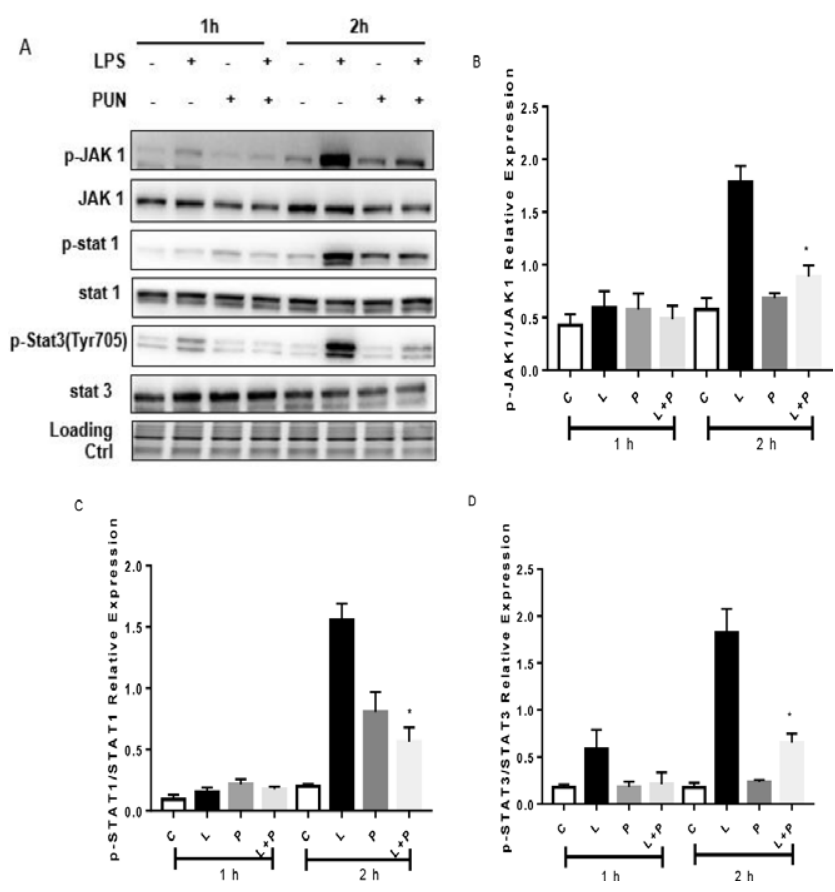

Figure 5: PUN inhibits LPS-induced activation of JAK/STAT signaling pathway in PMs Cells. Phosphorylation levels of JAK1, STAT1 and STAT3, as well as levels of SOCS $_{3}$ and SHP in PMs treated with $10 \mu \mathrm{g} / \mathrm{ml}$ LPS and $50 \mu \mathrm{M}$ PUN for the indicated time points. ${ }^{*} p<0.05$ compared with the LPS-treated group.

\section{DISCUSSION}

PUN accounts for about $10 \%$ of the tannin content of pomegranates $^{16}$ and has established anti-inflammatory effects. It inhibits the inhalable particulate matter (PM10)induced adhesion of monocytes to endothelial cells in vitro. In addition, both pomegranate peel extract and PUN reduce the inflammatory response caused by particulate matter. ${ }^{17}$ Studies ${ }^{18,19}$ show that PUN also significantly mitigates LPS-induced neuroinflammation, oxidative stress and memory impairment by inhibiting nuclear factor-kappa (NFx)-B.

Macrophages are the primary effectors of inflammation as well as tissue regeneration post bacterial infection through the production of pro- and anti-inflammatory cytokines respectively. ${ }^{20} \mathrm{~A}$ previous study has shown the anti-inflammatory action of PUN on the RAW 264.7 macrophage cell line. ${ }^{21}$ Consistent with this, we detected a significant decrease in the expression levels of iNOS, IL-1b, IL-6, IL-12p40, IL-12p70 and CCL-2, which are the hallmarks of activated macrophages, ${ }^{22,23}$ in the LPSstimulated primary PMs.

The MAPK pathway aggravates the inflammatory response by inducing production of effect or cytokines. MAPKs are serine/threonine protein kinases that are involved in

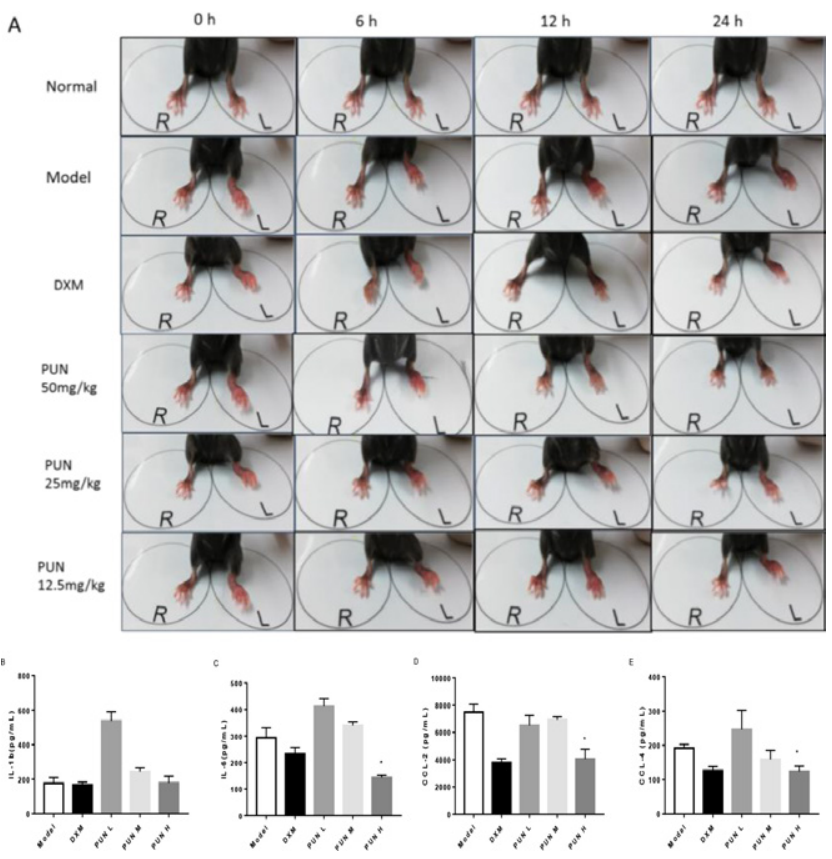

Figure 6: Effects of PUN in a zymosan-induced paw edema model. Edema was induced in the left hind paw of mice by a single injection of $50 \mu \mathrm{l}$ Zymosan (1\%), followed by administration of $12.5,50$ and $100 \mathrm{mg} / \mathrm{kg}$ PUN or $5 \mathrm{mM}$ dexamethasone (positive control) after $4 \mathrm{~h}$. A. Representative images showing paw edema in the indicated groups at different time points.

B-E. IL-1b, IL-6, CCL-2 and CCL-4 levels in the paw tissue homogenates of different groups. ${ }^{*} p<0.05$ compared with the model group. 
most cellular responses. ${ }^{24,25} \mathrm{PUN}$ inhibited phosphorylation of ERK in the LPS-challenged macrophages without affecting that of JNK and P38. The JAK/STAT pathway is frequently deregulated in various diseases and its constitutive activation is the basis of autoimmune diseases such as rheumatoid arthritis and ulcerative colitis. ${ }^{26,27}$ Binding of LPS to its receptor activates the receptorassociated JAK., which then leads to phosphorylation and nuclear translocation of STAT. The latter binds to the promoter region of its target genes, including those encoding for pro-inflammatory mediators. ${ }^{28-30}$ The JAK/ STAT pathway is regulated by $\operatorname{SOCS}^{31-33}$ that forms an inhibitory feedback loop by directly counteracting JAK and STAT activation. SHP2 is another inhibitor of this pathway that blocks the activity of JAKs. PUN not only inhibited JAK1, STAT1 and STAT3 phophorylation, but also slightly activated SOCS3 increasing its level.

The in vivo effect of PUN was finally assessed in a zymosaninduced model of acute inflammation. High dose PUN alleviated paw edema and also decreased the levels of pro-inflammatory cytokines in the paw homogenates. Taken together, PUN is a suitable candidate drug for inflammation-related diseases and future studies should focus on its clinical potential.

\section{CONCLUSION}

PUN inhibits secretion of pro-inflammatory cytokines from macrophages by suppressing the ERK/MAPK and JAK/STAT signaling pathways.

\section{ACKNOWLEDGEMENT}

The study was supported by Xinjiang Uygur Autonomous Region Key Laboratory Project of China (Grant No. 2016D03026) and the Open Fund of State Key Laboratory of Trauma, Burns and Combined Injury (No. SKLKF201605).

\section{CONFLICT OF INTEREST}

We declare that all authors have read and agreed to the submitted version of the paper and all who have been acknowledged as contributors have agreed to their inclusion. The material of this manuscript is original and it has not been published and neither under consideration for publication elsewhere. If the manuscript is accepted, the paper will not be published elsewhere in the same form, in English or in any other language, without written consent of the copyright holder. In addition, all the authors have no conflicts of interest to disclose.

\section{ABBREVIATIONS}

LPS: lipopolysaccharide; PUN: Punicalagin; PMs: peritoneal macrophages; MAPK: mitogen-activated protein kinase; JNK: c-Jun amino terminal kinase; ERK: extracellular protein kinase.

\section{REFERENCES}

1. Shao J, Wang P, Liu A, Du X, Bai J, Chen M. Punicalagin prevents hypoxic pulmonary hypertension via anti-oxidant effects in rats. J Am J Chin Med. 2016;44(4):785-801.

2. Foss SR, Nakamura CV, Ueda-Nakamura T, Cortez DA, Endo EH, Dias FBP. Antifungal activity of pomegranate peel extract and isolated compound punicalagin against dermatophytes. J Ann Clin Microbiol Antimicrob. 2014;13(4):32-4

3. Danesi F, Ferguson LR. Could pomegranate juice help in the control of inflammatory diseases. J Nutrients. 2017;9(9):909-24.

4. Liang YJ, Yang WX. Kinesins in MAPK cascade: How kinesin motors are involved in the MAPK pathway?. J Gene. 2019;684:1-9.

5. Biswas SK, Mantovani A. Macrophage plasticity and interaction with lymphocyte subsets: Cancer as a paradigm. J Nat Immunol. 2010;11(10):889-96.

6. Mosser DM, Edwards JP. Exploring the full spectrum of macrophage activation. Nat Rev Immunol J. 2008;8(12):958-69.

7. Ben SLA, Kim KH, Quah CC, Kim WR, Shahimi M. Anti-inflammatory potential of ellagic acid, gallic acid and punicalagin A andamp; B isolated from Punica granatum. J BMC Complement Altern Med. 2017;17(1):47.

8. Xu X, Yin P, Wan C, Chong X, Liu M, Cheng P, et al. Punicalagin inhibits inflammation in LPS-induced RAW264.7 macrophages via the suppression of TLR4-mediated MAPKs and NF-kappaB activation. J Inflammation. 2014;37(3):956-65.

9. Lachapelle AM, Ruygrok ML, Toomer M, Oost JJ, Monnie ML, Swenson JA, et al. The hormonal herbicide, 2, 4-dichlorophenoxyacetic acid, inhibits Xenopus oocyte maturation by targeting translational and post-translational mechanisms. J Reprod Toxicol. 2007;23(1):20-31.

10. Kim EK, Choi EJ. Pathological roles of MAPK signaling pathways in human diseases. J Biochim Biophys Acta. 2010;1802(4):396-405.

11. De S, Manna A, Kundu S, DeSarkar S, Chatterjee U, Sen T, et al. Allylpyrocatechol Attenuates Collagen-Induced Arthritis via Attenuation of Oxidative Stress Secondary to Modulation of the MAPK, JAK/STAT and Nrf2/ HO-1 Pathways. J Pharmacol Exp Ther. 2017;360(2):249-59.

12. Ci X, Ren R, Xu K, Li H, Yu Q, Song Y, et al. Schisantherin A exhibits antiinflammatory properties by down-regulating NF-kappaB and MAPK signaling pathways in lipopolysaccharide-treated RAW 264.7 cells. J Inflammation. 2010;33(2):126-36.

13. Li KK, Shen SS, Deng X, Shiu HT, Siu WS, Leung PC, et al. Dihydrofisetin exerts its anti-inflammatory effects associated with suppressing ERK/p38 MAPK and Heme Oxygenase-1 activation in lipopolysaccharide-stimulated RAW 264.7 macrophages and carrageenan-induced mice paw edema. J Int Immunopharmacol. 2018;54:366-74.

14. Li YY, Huang SS, Lee MM, Deng JS, Huang GJ. Anti-inflammatory activities of cardamonin from Alpinia katsumadai through heme oxygenase-1 induction and inhibition of NF-kappaB and MAPK signaling pathway in the carrageenaninduced paw edema. J Int Immunopharmacol. 2015;25(2):332-9.

15. DeOliveira FF, DeAraujo JC, Pereira AF, Brito GA, Gondim DV, Ribeiro RA et al. Antinociceptive and anti-inflammatory effects of Caryocar coriaceum Wittm fruit pulp fixed ethyl acetate extract on zymosan-induced arthritis in rats. J Ethnopharmacol. 2015;174:452-63.

16. Haidari M, Ali M, Ward C, Madjid M. Pomegranate (Punica granatum) purified polyphenol extract inhibits influenza virus and has a synergistic effect with oseltamivir. J Phytomedicine. 2009;16(12):1127-36.

17. Park S, Seok JK, Kwak JY, Suh HJ, Kim YM, Boo YC. Anti-Inflammatory Effects of Pomegranate Peel Extract in THP-1 Cells Exposed to Particulate Matter PM10. Evid Based Complement Alternat Med. 2016;6836080.

18. Kim YE, Hwang CJ, Lee HP, Kim CS, Son DJ, Ham YW, Hellstrom M, et al. Inhibitory effect of punicalagin on lipopolysaccharide-induced 
neuroinflammation, oxidative stress and memory impairment via inhibition of nuclear factor-kappa B. J Neuropharmacology. 2017;117:21-32.

19. Olajide OA, Kumar $A$, Velagapudi $R$, et al. Punicalagin inhibits neuroinflammation in LPS-activated rat primary microglia [J]. Molecular Nutrition and Food Research. 2015;58(9):1843-51.

20. Koh YC, Yang G, Lai CS, Weerawatanakorn M, Pan MH. Chemopreventive Effects of Phytochemicals and Medicines on M1/M2 Polarized Macrophage Role in Inflammation-Related Diseases. J Int J Mol Sci. 2018;19(8):2208.

21. Xu X, Yin P, Wan C, Chong X, Liu M, Cheng P, Chen J, et al. Punicalagin inhibits inflammation in LPS-induced RAW264.7 macrophages via the suppression of TLR4-mediated MAPKs and NF-kappaB activation. J Inflammation. 2014;37(3):956-65.

22. Szliszka E, Skaba D, Czuba ZP, Krol W. Inhibition of inflammatory mediators by neobavaisoflavone in activated RAW264.7 macrophages. J Molecules. 2011;16(5):3701-12

23. Guo D, Li JR, Wang Y, Lei LS, Yu CL, Chen NN. Cyclovirobuxinum D suppresses lipopolysaccharide-induced inflammatory responses in murine macrophages in vitro by blocking JAK-STAT signaling pathway. J Acta Pharmacol Sin. 2014;35(6):770-8.

24. Waetzig V, Czeloth K, Hidding U, Mielke K, Kanzow M, Brecht S, Goetz M, et al. C-Jun N-terminal kinases (JNKs) mediate pro-inflammatory actions of microglia. J Glia. 2005;50(3):235-46.

25. Bhat NR, Zhang P, Lee JC, Hogan EL. Extracellular signal-regulated kinase and p38 subgroups of mitogen-activated protein kinases regulate inducible nitric oxide synthase and tumor necrosis factor-alpha gene expression in endotoxin-stimulated primary glial cultures. J J Neurosci. 1998;18(5):1633-41.
26. Ghoreschi K, Laurence A, O'Shea JJ. Janus kinases in immune cell signaling J. Immunol Rev. 2009;228(1):273-87.

27. Roskoski RJ. Janus kinase (JAK) inhibitors in the treatment of inflammatory and neoplastic diseases. J Pharmacol Res. 2016;111:784-803.

28. Zhou Y, Wang J, Yang W, Qi X, Lan L, Luo L, Yin Z. Bergapten prevents lipopolysaccharide-induced inflammation in RAW264.7 cells through suppressing JAK/STAT activation and ROS production and increases the survival rate of mice after LPS challenge. J Int Immunopharmacol. 2017;48:159-68.

29. Kim HY, Park EJ, Joe EH, Jou I. Curcumin suppresses Janus kinaseSTAT inflammatory signaling through activation of Src homology 2 domain-containing tyrosine phosphatase 2 in brain microglia. J J Immunol. 2003;171(11):6072-9.

30. Kimura A, Naka T, Muta T, Takeuchi O, Akira S, Kawase I, et al. Suppressor of cytokine signaling-1 selectively inhibits LPS-induced IL-6 production by regulating JAK-STAT. J Proc Natl Acad Sci U S A. 2005;102(47):17089-94.

31. Babon JJ, Kershaw NJ, Murphy JM, Varghese LN, Laktyushin A, Young SN, et al. Suppression of cytokine signaling by SOCS $_{3}$ : Characterization of the mode of inhibition and the basis of its specificity. J Immunity. 2012;36(2):239-50.

32. Kershaw NJ, Murphy JM, Liau NP, Varghese LN, Laktyushin A, Whitlock $\mathrm{EL}$, et al. $\mathrm{SOCS}_{3}$ binds specific receptor-JAK complexes to control cytokine signaling by direct kinase inhibition. J Nat Struct Mol Biol. 2013;20(4):469-76.

33. Trengove MC, Ward AC. SOCS proteins in development and disease. J Am J Clin Exp Immunol. 2013;2(1):1-29.

Cite this article: Yan H, Gulimire A, Zhu J, Liang H, yi, Sr ZG. Punicalagin Suppresses LPS-induced Inflammatory Responses in Murine Macrophages via JAK/STAT Signaling Pathway and Zymosan-induced Mice Paw Edema. Indian J of Pharmaceutical Education and Research. 2021;55(2):550-5. 